\title{
TEXTURE CLASSIFICATION VIA PATCH-BASED SPARSE TEXTON LEARNING
}

\author{
Jin Xie, Lei Zhang, Jane You and David Zhang \\ Dept. of Computing, The Hong Kong Polytechnic University, Hong Kong, China \\ Email: \{csjxie, cslzhang, csyjia, csdzhang\}@comp.polyu.edu.hk
}

\begin{abstract}
Texture classification is a classical yet still active topic in computer vision and pattern recognition. Recently, several new texture classification approaches by modeling texture images as distributions over a set of textons have been proposed. These textons are learned as the cluster centers in the image patch feature space using the $K$-means clustering algorithm. However, the Euclidian distance based the $K$-means clustering process may not be able to well characterize the intrinsic feature space of texture textons, which if often embedded into a low dimensional manifold. Inspired by the great success of $l_{1}$-norm minimization based sparse representation (SR), in this paper we propose a novel texture classification method via patch-based sparse texton learning. Specifically, the dictionary of textons is learned by applying SR to image patches in the training dataset. The SR coefficients of the test images over the dictionary are used to construct the histograms for texture classification. Experimental results on benchmark database validate the effectiveness of the proposed method.
\end{abstract}

Index Terms - Texture classification, texton, sparse representation, $K$-means

\section{INTRODUCTION}

Texture classification is an important research topic in computer vision and pattern recognition applications, such as image understanding and object recognition, and texture classification has been receiving considerable attention over the past decades. The co-occurrence matrix [1], which exploits the non-parametric statistics at the pixel level, is still a popular texture classification approach. The polar coordinate system has been used for rotation invariant texture classification [2]. The Local Binary Pattern (LBP) proposed by Ojala et al. [3] has become a benchmark method in rotation invariant texture classification.

However, the above traditional methods are sensitive to changes in viewpoint. Some recent approaches have been proposed to solve this problem. Lazebnik et al. [4] used invariant descriptors defined on affine invariant regions to describe texture features. $\mathrm{Xu}$ et al. [5] proposed the multifractal spectrum vectors to describe textures while achieving global invariance. Leung and Malik [6] proposed to classify texture images by using three dimensional (3D) textons, which are cluster centers of filter responses over a stack of images with representative viewpoints and illuminations. Varma and Zisserman [7, 8] modeled texture images as distributions over a set of textons, which are learned from the responses of MR8 filter banks [7]. Furthermore, in [9, 10], better performance was obtained by using textons learned from patches in the original image instead of MR8 filter responses.

In these texton based methods, the textons are usually learned by the $K$-means clustering algorithm. However, the $K$-means clustering algorithm is based on the $l_{2}$-norm Euclidean distance so that the elements of a cluster will have a ball-like distribution. The learned $K$ ball-like clusters, nonetheless, may not be able to characterize reasonably well the intrinsic feature space of the texture images, which is often embedded into a lower dimensional manifold.

Recently, the theory and algorithms of sparse coding or sparse representation (SR) $[11,12]$ have been successfully used in image processing and pattern recognition [13, 14]. The principle of SR reveals that a given natural signal can be often sparsely represented as the linear combination of an over-complete dictionary via $l_{1}$-norm minimization $[12,15]$. Inspired by the great success of SR and patch based texton learning $[9,10]$, in this paper we propose a patch based sparse texton learning method for texture classification. The idea of learning a dictionary of atoms from the training samples under the SR framework, and then use the learned dictionary to represent the testing samples has been recently successfully used in face recognition [18, 19]. A texton training dataset is first constructed by extracting patches in the training images, and then an over-complete dictionary of patch textons is learned from it under the SR framework. By sparsely representing the texture image over the learned texton dictionary, a histogram of SR coefficients can be computed and used as features for texture classification. It will be seen that the proposed method can achieve better texture classification performance than state-of-the-art texton based texture classification method using textons learned by $K$-means clustering.

The rest of the paper is organized as follows. Section 2 briefly reviews the concepts of SR. Section 3 describes in detail the proposed patch-based texton learning and texture classification scheme. Section 4 presents experimental results and Section 5 concludes the paper. 


\section{SPARSE REPRESENTATION OF SIGNALS}

In recent years there has been a growing interest in the study of SR of signals. The success of SR largely owes to the fact that natural signals are intrinsically sparse in some domain. Therefore, if the dictionary that is used to define the sparse domain can be well trained, a good analysis of the input signal can be expected by representing over the dictionary.

For a given signal $x \in R^{m}$, we say that $x$ has a sparse approximation over a dictionary $D=\left[\boldsymbol{d}_{1}, \boldsymbol{d}_{2}, \ldots, \boldsymbol{d}_{l}\right] \in R^{m \times l}$, if we can find a linear combination of only "a few" atoms from $D$ that is "close" to the signal $x$. Under this assumption, the sparsest representation of $x$ over $D$ is the solution of

$$
\arg \min _{\alpha}\|\boldsymbol{\alpha}\|_{p} \text { s.t. }\|x-D \boldsymbol{\alpha}\|_{2} \leq \varepsilon
$$

where $\|\boldsymbol{\alpha}\|_{p}$ is a sparsity-inducing regularization term.

In some applications, the dictionary $D$ is unknown and we need to learn it from a training dataset $X=\left[\boldsymbol{x}_{1}, \boldsymbol{x}_{2}, \ldots, \boldsymbol{x}_{n}\right] \in$ $R^{m \times n}$. It is expected that each training sample (i.e. each column of $X$ ) can be sparsely represented over the dictionary $D$, i.e. $\boldsymbol{x}_{i}=D \boldsymbol{\alpha}_{i}$ and only a few elements in $\boldsymbol{\alpha}_{i}$ are significant. The dictionary $D$, as well as the SR coefficient vectors $\boldsymbol{\alpha}_{i}$, can be solved by optimizing the following objective function

$$
\arg \min _{D,\left\{\alpha_{i}\right.} \sum_{i=1}^{n}\left\|\boldsymbol{\alpha}_{i}\right\|_{p} \text { s.t. }\|X-D \Lambda\|_{F}^{2} \leq \varepsilon
$$

where $\Lambda=\left[\boldsymbol{\alpha}_{1}, \boldsymbol{\alpha}_{2}, \ldots, \boldsymbol{\alpha}_{n}\right]$ and $\|\bullet\|_{F}$ is the Frobenius matrix norm.

\section{PATCH-BASED TEXTON LEARNING FOR TEXTURE CLASSIFICATION}

In this section, we propose to learn the dictionary of textons under the SR framework, and then use the SR coefficients of a texture image over the learned texton dictionary to classify the texture images. In our work, the textons are learned from the original image patches. Therefore, in the following sub-sections, we briefly describe the pre-processing for training dataset construction, and then present the details of sparse texton learning and texture classification.

\subsection{Patch-based texton learning}

Before texton learning, all training texture images are converted to grey level images and are normalized to have zero mean and unit standard deviation. The normalization offers certain amount of invariance to the illumination changes. A square neighborhood around each pixel in the image is cropped and is stretched to a vector. All the patch vectors are contrast normalized using Weber's law. Hence, for each class of texture images, we can construct a training dataset $X=\left[\boldsymbol{x}_{1}, \boldsymbol{x}_{2}, \ldots, \boldsymbol{x}_{n}\right]$, where $\boldsymbol{x}_{i}, \mathrm{i}=1,2, \ldots, n$, is the patch vector at a position in a training sample image of this class.

The dictionary of textons, denoted by $D=\left[\boldsymbol{d}_{1}, \boldsymbol{d}_{2}, \ldots, \boldsymbol{d}_{l}\right]$, can be learned from the constructed training dataset $X$, where $\boldsymbol{d}_{j}, j=1,2, \ldots, l$, is one of the $l$ textons. In $[9,10]$, the classical $K$-means clustering method was employed to determine the $l$ textons by solving the following optimization problem:

$$
\arg \min _{D} \sum_{j=1}^{l} \sum_{\boldsymbol{x}_{k} \in \Omega_{j}}\left\|\boldsymbol{x}_{k}-\boldsymbol{d}_{j}\right\|_{2}^{2}
$$

Obviously, the $K$-means clustering will partition the dataset $X$ into $l$ groups $\Omega_{1}, \ldots, \Omega_{j}, \ldots, \Omega_{l}$, and the texton $\boldsymbol{d}_{j}$ is defined as the mean vector of the vectors within $\Omega_{j}$. However, as we explained in the Introduction, by using $K$-means clustering, the elements belong to the same cluster will distribute within a ball because the $l_{2}$-norm Euclidean distance is used in the clustering process. These ball-like clusters should cover the whole feature space. Nonetheless, such a dense coverage may not be able to effectively characterize the intrinsic feature space of texture images, which is often embedded into a lower dimensional manifold.

Let $\Lambda=\left[\boldsymbol{\alpha}_{1}, \boldsymbol{\alpha}_{2}, \ldots, \boldsymbol{\alpha}_{n}\right]$, the SR objective function in Eq. (2) is adopted to optimize $D$ and $\left\{\boldsymbol{\alpha}_{i}\right\}$, and here we re-write it as follows by setting $p=1$ :

$$
\arg \min _{D, \Lambda}|\Lambda|_{1} \text { s.t. }\|X-D \Lambda\|_{F}^{2} \leq \varepsilon
$$

In practice, it is more convenient to convert Eq. (4) into an unconstrained optimization problem by using a form of $l_{1^{-}}$ penalized least-squares:

$$
\arg \min _{D, \Lambda}\|X-D \Lambda\|_{F}^{2}+\lambda\|\Lambda\|_{1}
$$

Eqs. (4) and (5) are equivalent with an appropriate parameter $\lambda$, which is used to balance the $l_{1}$-norm and $l_{2}$ norm terms in Eq. (5). In this paper, we adopted the recently proposed feature-sign search method [16] to solve Eq. (5) because this algorithm is more efficient.

In some sense, the $K$-means clustering method can be viewed as a special case of the SR based clustering in Eq. (5). If we let $\boldsymbol{\alpha}_{i}$ has only one non-zero element and let this non-zero element be 1 , then Eq. (5) will be basically the same as Eq. (3). In this case, we use only one texton to represent the feature vector $\boldsymbol{x}_{i}$ and assign the label of $\boldsymbol{x}_{i}$ to that texton. In contrast, by using SR, $\boldsymbol{x}_{i}$ or any input vector $\boldsymbol{y}$ will be coded as a linear combination of more than one textons. Therefore, SR can achieve a much lower reconstruction error due to the less restrictive constraint. In addition, for an input vector $\boldsymbol{y}$ which may lie in the boundary of two or more clusters, the $K$-means clustering will randomly assign it to one of the classes. Such a representation may not be efficient enough in practice. By using the proposed SR based method, such problem can be avoided because the boundary samples will be represented by multiple textons. In the experiments in Section 4, we will see that by using Eq. (5) to learn the textons and using the 
associated feature description method in Section 3.2, the texture classification accuracy can be improved.

\subsection{Feature description and texture classification}

Denote by $D_{k}$ the learned texton dictionary for the $k^{\text {th }}$ texture class, the dictionary for all the $c$ classes of texture images can be formed by amalgamating the $K$ dictionaries

$$
D=\left[D_{1}, D_{2}, \ldots, D_{c}\right]
$$

With this dictionary $D$, each training texture image can generate a model by mapping it to the texton dictionary. In Varma and Zisserman's method [9, 10], for each position of a training image, it is labeled with the elements in the texton dictionary $D$ that is closest to the image patch vector at this position. Therefore, a histogram can be formed by normalizing the frequencies of texton labels of this image.

Different from the method in $[9,10]$, we can construct a histogram of the SR coefficients of a training image as the texture model. Denote by $\boldsymbol{x}_{i}$ the image patch vector at position $i$ of a training image, we can represent $\boldsymbol{x}_{i}$ over $D$ by $\mathrm{SR}$ to get the representation coefficient vector. However, this can be computationally very expensive because $D$ can be very big. Let $D=\left[\boldsymbol{d}_{1}, \boldsymbol{d}_{2}, \ldots, \boldsymbol{d}_{z}\right]$, where $z$ is the total number of textons learned from the $c$ classes. In practice, we can use only a subset of $D$ to represent $\boldsymbol{x}_{i}$. Specifically, we use the closest $t$ textons $(t<<z)$ to $\boldsymbol{x}_{i}$ in $D$ to form the sub-dictionary for $\boldsymbol{x}_{i}$. Denote by $\boldsymbol{d}_{1}^{i}, \ldots, \boldsymbol{d}_{t}^{i}$ the $t$ closest textons to $\boldsymbol{x}_{i}$, the subdictionary for $\boldsymbol{x}_{i}$ is then $D_{i}=\left[\boldsymbol{d}_{1}^{i}, \ldots, \boldsymbol{d}_{t}^{i}\right]$. The representation vector of $\boldsymbol{x}_{i}$ over $D_{i}$, denote $\boldsymbol{\alpha}_{i}=\left[\boldsymbol{\alpha}_{1}^{i}, \ldots, \boldsymbol{\alpha}_{t}^{i}\right]$, can then be computed by solving he following $l_{1}$-norm minimization problem:

$$
\arg \min _{\boldsymbol{\alpha}_{i}}\left\|\boldsymbol{x}_{i}-D_{i} \boldsymbol{\alpha}_{i}\right\|_{2}^{2}+\lambda\left\|\boldsymbol{\alpha}_{i}\right\|_{1}
$$

The $l_{1}$-least square method in [10] can be used to solve Eq. (7).

Since the textons $\boldsymbol{d}_{1}^{i}, \ldots, \boldsymbol{d}_{t}^{i}$ in $D_{i}$ have a one-to-one correspondence to the textons in $D$, by using $\alpha_{i}$ we can easily construct another representation vector $\boldsymbol{h}_{i}$ of $\boldsymbol{x}_{i}$ over $D$ such that

$$
D_{i} \boldsymbol{\alpha}_{i}=D \boldsymbol{h}_{i}
$$

Obviously, most of the entries in $\boldsymbol{h}_{i}$ will be 0 , and only the entries corresponding to the same textons as those in $D_{i}$ will have non-zeros values, and these values are the same as those in $\alpha_{i}$.

Finally, at each position $i$ of a training texture image, we have a representation vector $\boldsymbol{h}_{i}$. Because the coefficients in $\boldsymbol{h}_{i}$ are real numbers instead of integers, we can form a fractional histogram, denoted by $H_{f}$, for this texture image by summing all the vectors of $\boldsymbol{h}_{i}$ :

$$
H_{f}=\sum \boldsymbol{h}_{i}
$$

The fractional histogram $H_{f}$ can serve as the texture model.

Denote by $H_{i}, i=1,2, \ldots, n$, the model histograms in the database. Similarly, for an input test image $Y$, we can construct the sparse texton histogram for it, denoted by $H_{y}$. The similarity between $H_{i}$ and $H_{y}$ is computed by:

$$
\chi^{2}\left(H_{\mathrm{i}}, H_{\mathrm{y}}\right)=\frac{1}{2} \sum \frac{\left(H_{\mathrm{i}}-H_{\mathrm{y}}\right)^{2}}{H_{\mathrm{i}}+H_{\mathrm{y}}}
$$

The texture image $Y$ is classified to the corresponding texture class by a nearest neighbor classifier.

\section{EXPERIMENTAL RESULTS}

In this section, we evaluate the proposed texture classification method on the CUReT database [17]. The CUReT texture database contains 61 classes, each consisting of 205 images. Here we choose 92 images per class for which a large region of texture is visible across all textures. There are a number of factors that make the CUReT texture database challenging. It has both large inter-class confusion and intra-class variation. The images of a class are obtained under unknown viewpoint and illumination, and some different classes look similar in appearance. Figs. 1(a) and (b) show two different kinds of textures while the images appear similar.
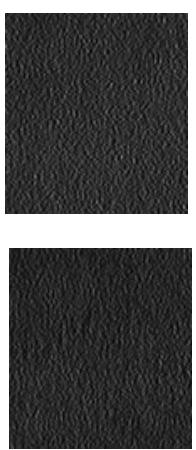

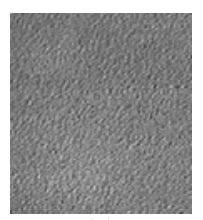

(a)

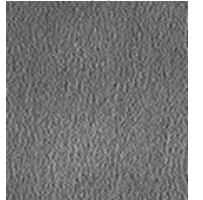

(b)
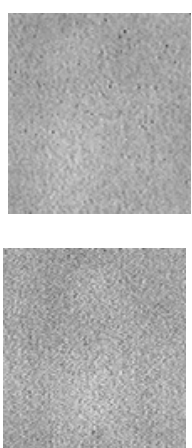

Figure1: Two different kinds of texture samples with similar appearance.

The evaluation methodology on the CUReT database is as follows: $M$ images are chosen per class for training and the remaining 92- $M$ images per class are used to form the test set. In the experiment, 46, 23, 12, and 6 texture images per class are chosen as the training set. Table 1 compares the performance of our proposed method with the state-of-theart patch texton based method proposed by Varma and Zisserman $[9,10]$ (we denote this method as VZ_Patch). For the VZ_Patch and the proposed method, a $9 \times 9$ neighborhood around each pixel was taken and an 81 dimensional feature vector was formed. 40, 30 and 20 textons per texture class were learned, which resulted in 2440, 1830 and 1220 dimensional histograms for texture representation respectively.

When $M=46$, the proposed method achieves the accuracies of $97.98 \%, 97.84 \%$ and $97.52 \%$ using 40,30 and 20 textons per class respectively, while the VZ_Patch method achieves the accuracies of $97.11 \%, 97.08 \%$ and $96.61 \%$. With the decrease of number of training samples, the proposed method can still achieve better recognition 
accuracy than the VZ_Patch method. Especially, when $M=6$, the improvement of the proposed method over the VZ_Patch method is more obvious. Note that in this experiment we did not compare our method with another two state-of-the-art methods, the Lazebnik's method [4] and Xu's method [5]. This is because on the CUReT texture database, the affine invariant detector cannot produce enough regions for a robust statistical characterization of the texture, while because of low resolution images in this database, the multifractal spectrum features used in [5] cannot be well extracted for classification.

\begin{tabular}{|c|c|c|c|}
\hline Algorithms & \multicolumn{3}{|c|}{46 training images per class } \\
\hline & 40 textons & 30 textons & 20 textons \\
\hline VZ_Patch & $97.11 \%$ & $97.08 \%$ & $96.61 \%$ \\
\hline proposed & $97.98 \%$ & $97.84 \%$ & $97.52 \%$ \\
\hline \multicolumn{4}{|c|}{ (a) } \\
\hline Algorithms & \multicolumn{3}{|c|}{23 training images per class } \\
\hline & 40 textons & 30 textons & 20 textons \\
\hline VZ_Patch & $95.34 \%$ & $95.06 \%$ & $94.96 \%$ \\
\hline proposed & $96.05 \%$ & $95.71 \%$ & $95.54 \%$ \\
\hline \multicolumn{4}{|c|}{ (b) } \\
\hline Algorithms & \multicolumn{3}{|c|}{12 training images per class } \\
\hline & 40 textons & 30 textons & 20 textons \\
\hline VZ_Patch & $91.23 \%$ & $90.88 \%$ & $90.47 \%$ \\
\hline proposed & $92.08 \%$ & $91.86 \%$ & $91.57 \%$ \\
\hline \multicolumn{4}{|c|}{ (c) } \\
\hline Algorithms & \multicolumn{3}{|c|}{6 training images per class } \\
\hline & 40 textons & 30 textons & 20 textons \\
\hline VZ_Patch & $84.56 \%$ & $84.06 \%$ & $83.38 \%$ \\
\hline proposed & $85.76 \%$ & $85.24 \%$ & $84.46 \%$ \\
\hline
\end{tabular}

Table1: Classification accuracies on the CUReT texture database using (a) 46; (b) 23; (c) 12 and (d) 6 training samples.

\section{CONCLUSIONS}

In this paper, we proposed to use the sparse representation (SR) technique to learn the texton dictionary for texture image representation, and then use the SR coefficients of an input image over the learned dictionary for feature description. A histogram of the SR coefficients is constructed for texture classification. Our experimental results on the CUReT texture database validated that the proposed method can achieve higher classification accuracy than the state-of-the-art texton learning based texture classification method. In future work, we will investigate how to further improve the classification accuracy and reduce the size of texton dictionary.

\section{ACKNOWLEDGEMENT}

This research is supported by the Hong Kong RGC General Research Fund (PolyU 5330/07E) and the Hong Kong PolyU Internal Fund (G-YH54).
[1] R.M.Haralick, K.Shanmugam, and I. Dinstein, "Textural features for image classification," IEEE Trans. Systems, Man and Cybernetics, vol. 3, pp. 610-621, 1973.

[2] Chi-Man Pun, and Moon-chuen Lee, "Log-polar wavelet energy signatures for rotation and scale invariant texture classification," IEEE TPAMI, vol. 26, pp. 1228-1333, 2004.

[3] T.Ojala, M.Pietikainen, and T.Maenpaa, "Multiresolution grayscale and rotation invariant texture classification with local binary patterns," IEEE TPAMI, vol. 24, pp. 971-987, 2004.

[4] S.Lazebnik, C.Schmid, and J.Ponce, "A sparse texture representation using local affine regions," IEEE TPAMI, vol. 27, pp. 1265-1278, 2005.

[5] Y.Xu, H.Ji, and C.Fermuller, "A projective invariant for textures," in Proceedings of Computer Vision and Pattern Recognition, IEEE, 2006, pp. 1932-1939.

[6] T.Leung, and J.Malik, "Representing and recognizing the visual appearance of materials using three-dimensional textons," International Journal of Computer Vision, vol. 43, pp. 29-44, 2001. [7] M.Varma, and A.Zisserman, "Classifying images of materials: achieving viewpoint and illumination independence," European Conference on Computer vision, IEEE, 2002, vol. 3, pp. 255-271.

[8] M.Varma, and A.Zisserman, "A statistical approach to texture classification from single images," International Journal of Computer Vision: Special Issue on Texture Analysis and Synthesis, vol. 62, pp. 61-81, 2005.

[9] M.Varma, and A.Zisserman, "Texture classification: are filter banks are necessary?" in Proceedings of Computer Vision and Pattern Recognition, IEEE, 2003, vol. 2, pp. 691-698.

[10] M.Varma, and A.Zisserman, "A statistical approach to material classification using image patch exemplars," IEEE TPAMI, vol. 31, pp. 2032-2047, 2009.

[11] D.Donoho, "For most large underdetermined systems of linear equations the minimal $l_{1}$-norm solution is also the sparsest solution," Communications on Pure \& Applied Mathematics, vol. 59, pp. 797-829, 2006.

[12] R.Tibshirani, "Regression shrinkage and selection via the lasso," Journal of Royal Statistical Society, vol. 58, pp. 267-288, 1996.

[13] J.Mairal, M. Elad, and G.Sapiro, "Sparse representation for color image restoration," IEEE Trans. Image Processing, Vol. 17, pp.53-69, 2008.

[14] John Wright, Allen Yang, Arvind Ganesh, Shankar Sastry, and Yi Ma, "Robust face recognition via sparse representation," IEEE TPAMI, vol. 31, 2009.

[15] S.S.Chen, and D.L.Donoho, and M.A.Saunders, "Atomic decomposition by basis pursuit," SIAM Review, vol. 43, pp.129159,2001

[16] H.Lee, A.Battle, R.Raina, and A.Y.Ng, "Efficient sparse coding algorithms," Advances in Neural Information Processing Systems, 2006.

[17] K.J.Dana, B.vanGinneken, S.K.Nayar, and J.J.Koenderink, "Refelctance and texture of real world surfaces," ACM Trans. Graphics, vol. 18, pp. 1-34, 1999.

[18] M. Yang and Lei Zhang, "Gabor Feature based Sparse Representation for Face Recognition with Gabor Occlusion Dictionary," in ECCV 2010.

[19] Meng Yang, Lei Zhang, Daivd Zhang and Jian Yang, "Metaface learning for sparse representation based face recognition," in ICIP 2010. 